\title{
State Estimation and Determination of Flexibility Potential in Medium Voltage Networks*
}

\author{
Florian Rewald ${ }^{\dagger}$, Oliver Pohl $^{1}$, Ulf Häger $^{1}$, and Christian Rehtanz ${ }^{1}$ \\ 1 TU Dortmund University, 44227 Dortmund, Germany
}

\begin{abstract}
This paper describes how flexibility of medium voltage systems can be used for operation of high voltage systems. This is explained using the example of a congestion management system for high voltage networks developed in the research project IDEAL. First, it is explained to what extent flexibility is required for high voltage system operation. After that, the entire congestion management system with flexibility of medium voltage systems is presented. Then, methodology for observability analysis, state estimation and determination of flexibility potential is shown. Finally, the implementation of the methodology in two real medium voltage systems is described.
\end{abstract}

\section{Introduction}

Due to climate change many countries try to limit the production of greenhouse gas emissions. In Germany the production of greenhouse emissions will be reduced by $85 \%$ until 2050 . This is one reason, why the energy system is aimed to be transformed sustainably and all coal fired power plants will be shut down by 2038 and even all nuclear power plants until 2022. The existing large fossil and nuclear power plants will be replaced by many small distributed energy resources (DER) and by large off shore wind farms.

This means not only a change of energy supply, it is also a change of the structure of the entire energy system. The large number of DER with small installed capacity is mostly connected to medium voltage (MV) or low voltage (LV) networks, which implies a shift of energy supply from transmission and sub-transmission systems to distribution systems. Off Shore wind farms feed into the transmissions networks as the most fossil power plants, but the distance to the load centres in West and South of Germany is much bigger than the distance between the fossil power plants and the industry regions toady.

Furthermore, the feed-in of photovoltaic (PV) power plants and wind farms is strongly weather-dependent. In addition, power lines and transformers are overloaded due to the lack of network expansion to this day. This leads to an increased need for balancing energy in the entire energy system.

This balancing energy is mostly provided by fossil power plants, which will be shut down in a few years. Therefore, DER must also provide flexibility in the future. This means a higher coordination effort between distribution system operator (DSO) and transmission system operator (TSO), which has not yet occurred. Furthermore, MV and LV networks are only designed for load cases and not for injection cases. LV networks are normally fixed coupled via a transformer to a MV network and not equipped with measurement devices for estimating system state. Finally, feasible flexible generation units are mostly not equipped with communication technology.

The use of flexibility in high voltage (HV) system operation requires an estimation of MV system state and an approach for determining flexibility potentials. Approaches for estimating system state considering specific requirements of distribution systems are presented in [1] or [2]. The optimal localization of measurement devires for estimating system state is analysed e.g. in [3]. The determination of flexibility potential of distr bution systems is addressed e.g. in [4], [5] or [6]. Thereby, it is often assumed that distribution systems are fully observable.

This paper presents a practical approach for determining flexibility potential of MV for HV system operation considering incomplete observability of MV systems. In this case flexibility means all feasible set points of MV systems at connecting point to HV system, which can be provided without violating any network restrictions. Set point of the distribution system can be changed by changing the set points of flexible generation units. Flexibility options are needed to provide the demand of flexibility in HV systems. In this case all DER connected to MV systems are considered as flexible generation units and all DER can provide flexibility.

\footnotetext{
*This work was supported by the German Federal Ministry for Economic Affairs and Energy (BMWi) in the course of the project IDEAL (FKZ: 03ET7557A).

${ }^{\dagger}$ Corresponding author: florian.rewald@tu-dortmund.de
} 
The main goal of this paper is to show how flexibility of distribution systems can be used for HV system operation. This is explained using the example of $\mathrm{HV}$ congestion management system (CMS) with flexibility of MV systems. This is part of research project Impedance Controllers and Decentralized Congestion Management for Autonomous Load Flow Control (IDEAL) 11. This project focuses on developing an agent-based $\mathrm{HV}$ congestion management system based on distributed power flow controller (DPFC) and flexibility of underlying MV and LV networks. For this reason, the flexibility potential of the distribution system is determined based on estimated system state. This paper is structured as follows: Section II gives an overview for the use of flexibility from distribution for transmission systems. After that, the entire CMS approach based on DPFC and flexibility of underlying distribution systems is explained in section III. Afterwards section IV gives an overview about estimating of system state and determination of flexibility potentials. Sections V focuses on the real implementation of the whole system in field test. The paper concludes with an outlook for further research questions.

\section{Medium Voltage System Flexibility for High Voltage Systems}

For the operation of an electrical power system flexible power is required for frequency stability as well as preventive and curative elimination of network bottlenecks. This section describes in which cases flexibility of MV and LV networks can be used for transmission and sub-transmission systems.

\subsection{Flexibility for Frequency Stability}

Energy supply and load must be balanced for a constant frequency. Within a control area, balancing occurs through trading on the electricity power exchange up to a maximum of five minutes before the energy is made available. Due to the increasing installed capacity of volatile PV and wind power plants as well as the stochastic behaviour of loads energy feed-in and load in control areas are not balanced in every situation. Therefore, operating reserve is used, which is mostly provided by fossil power plants. Due to the shut down of these power plants, one option for providing operating reserve is the use of flexibility of distribution systems.

\subsection{Flexibility for preventive Elimination of Bot- tlenecks}

Flexibility is also used preventively to minimize potential bottle necks in transmission and subtransmission systems. Therefore, the injection of two power plants between which the bottleneck is located are increased or respectively decreased. According to the law redispatch is carried out by power plants whose installed capacity is more than $50 \mathrm{MW}$.

\subsection{Flexibility for curative Elimination of Bottle- necks}

To eliminate existing bottlenecks and violations of voltage restrictions flexibility of distribution systems can also used in same voltage level as well[7]. In this case it is allowed to regulate loads and injections of DER. This regulated power increased in the last few years and is likely to rise further since the use of load shifting for the network planing process [8]. This means that distribution networks are not designed for all high feed-in peaks. So the feed-in management becomes a tool for the system operation process and the need for flexibility increase.

\subsection{Research Questions}

From the perspective of MV and LV system operator the following questions have to be addressed:

- In which cases can flexibility of MV systems be used for system operation of HV system?

- How can system state in a MV network be estimated?

- How can the flexibility potential be determined based on estimated system state?

- What can a CMS look like across both voltage levels?

- What influence do measurement inaccuracies and large measurement errors have on the system state?

- How can pseudo measurements be determined based on metro logically developed substations for substations without measurement devices?

- How can the injection and load at substations be forecast depending on the weather?

- Can an ideal number and placement of measurement devices be derived from the previous investigations?

The main goal of this paper is to give an overview of the CMS from the perspective of DSO. The aim is to describe how the questions described above can be answered within the framework of the project, not giving an answer to all these questions.

\footnotetext{
${ }^{1}$ Original name in German: Impedanzregler und Dezentrales Engpassmanagement für Autonome Lastflussregelung
} 


\section{Congestion Management System with Flexibility of Distribution Systems}

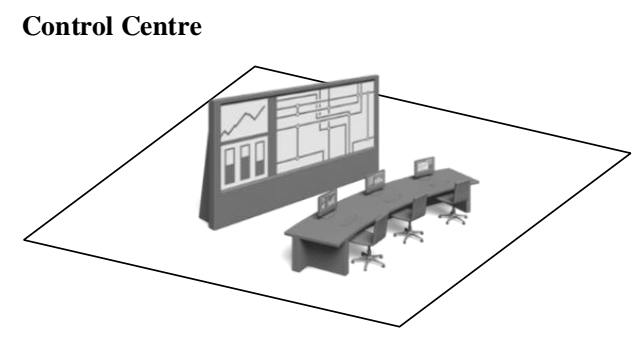

HV

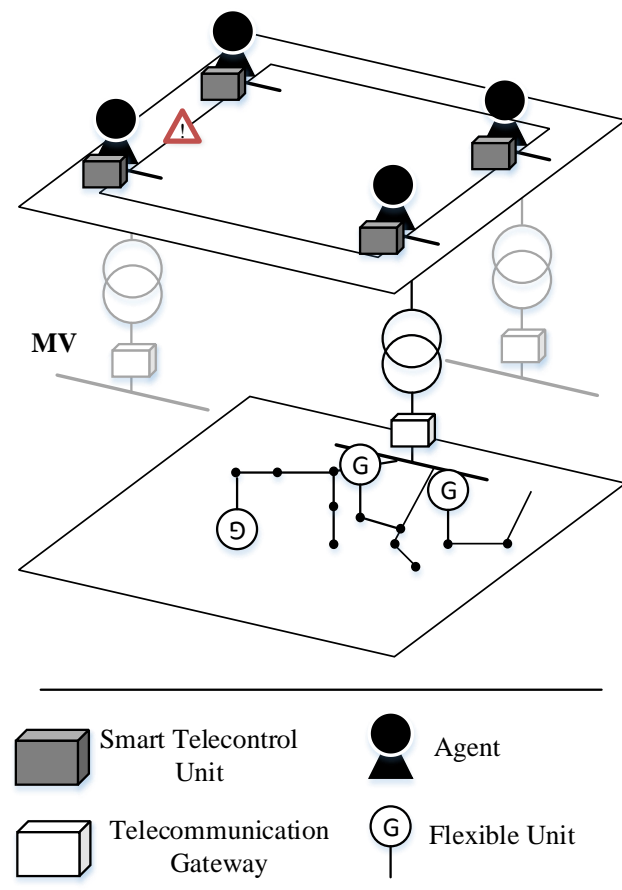

Figure 1: Overview of developed CMS [9]

The main goal of the research project IDEAL is to develop an agent-based congestion management systems for HV system with DPFC and flexibility of underlying distribution systems. An overview of the whole system is visualized in figure (1). The figure consists of the levels control centre, HV and MV systems is intended to explain the components of the system and elimination of the shown bottleneck. A detailed description can be found in 9 .

\subsection{Distributed Power Flow Controller}

The used DPFC are so called Distributed Series Reactors (DSRs). These devices consists of a coil and have the capability of injecting inductance or capacitance into the power line. So the reactance of power lines can increase or decrease and power flow can be controlled in a meshed system to eliminate bottleneck. These devices are directly clipped on the overhead power line. First installation and field tests of DSR have been already done in 2012. If the bottleneck cannot be eliminated by DPFC flexibility of underlying MV systems will be used.

\subsection{Flexibility of Medium Voltage Systems}

Flexibility of MV systems can be used for eliminating existing bottlenecks in HV system. At the request of the HV system operator, MV systems operator has to change the active and reactive power flow at the connection point between HV and MV systems. Thereby, network restrictions of MV and LV systems have not been violated. The methodology of determining system state, flexibility potential and implementation in field test are described in sections IV, V and VI.

\subsection{Hardware Components}

To coordinate the DPFC and the use of flexibility potentials hardware devices are used. Smart Telecontrol Unit (STU) are installed at every transformer station. On these devices the multi agent system (MAS) for agent communication is hosted. Furthermore, every flexible MV system is equipped by Telecontrol Gateway (TCG). On these gateways, flexibility potential is calculated based on incoming measurement values.

\subsection{Centralized and Decentralized Control Strate- gies}

Due to the high number of decentralized units in further energy systems, decentralized and autonomous control strategies based on MAS are used for the proposed CMS. MAS gives just a recommendation to control centre for eliminating the existing bottlenecks. Due to high security requirements for system operation and for research purposes, MAS is also implemented in control centre to compare results of MAS and control centre for eliminating bottlenecks.

\subsection{Information Flow and Decision Making}

An agent is implemented on a STU in every $\mathrm{HV} / \mathrm{MV}$ transformer station. The power lines and flexible MV systems are assigned to one agent. The agent gets voltage and current measurement of assigned power lines with DPFC and flexibility potentials of assigned MV systems. Agents share status information to each other at every time. In case of overloaded line assigned agent inform the other agents. Now every agent determines a negotiation of actions for eliminating network bottleneck and sends it to the assigned agent, which decide for one negotiation of action. During autonomous mode agent executes the best negotiation of action, otherwise it sends the recommendation to control centre and waits for further introductions.

\section{Estimation of System State and Deter- mination of Flexibility Potential}

For an active participation of MV systems in eliminating HV system bottlenecks with the use of flexibility determination of system is necessary. Estimation of system state is possible if the system is observable. This section describes the approaches for analysis of observability, estimation of system state and determination of flexibility potential. 


\subsection{Observability Analysis}

To Estimate system state a sufficient number of measured values as well as a homogenous location in the system is required. Observability analysis determines, whether the number and location of the available measured values in a network is sufficient for the calculation of a complex voltage at every single node. The observability can be expressed as a non-linear vector equation $h_{o b s}$ depending on measurement vector $z$ and system state vector $x_{o b s}$ as shown in (1). 10

$$
h_{o b s}\left(x_{o b s}\right)=z
$$

Thereby, vector $x_{\text {obs }}$ consists of branch voltage and phase angle variables $\alpha_{1}, \alpha_{2}$ and $\alpha_{l}$ as well as $\delta_{1}, \delta_{2}$ and $\delta_{l} . \quad l$ is the number of branches. These variables are determined by (2) and (3) for any branch $j$.

$$
\begin{gathered}
\alpha_{j}=\ln \frac{V_{k}}{V_{m}} \\
\delta_{j}=\theta_{k}-\theta_{m}
\end{gathered}
$$

Thereby, $k$ and $m$ are nodes which branch $j$ connected. In the next step (1) is approximated with the Taylor series in (4).

$$
H_{o b s} \cdot \Delta x=z-f_{o b s}\left(x^{0}\right)=\Delta z
$$

where $H_{o b s}=\frac{\partial h_{o b s}\left(x_{o b s}\right)}{\partial x_{o b s}}$ and $\Delta x_{o b s}=x_{o b s}-x_{o b s}^{0}$. The Jacobian matrix consists of branch variables and measurement equations. Measurement equations contain all measurement values expressed as non linear function of branch variables of voltage $\alpha$ and phase angles $\delta$.

Jacobian matrix is decomposed into its triangular factors with Peters-Wilkinson method. Zero pivots of upper triangular matrix are substituted by 1.0. This is equivalent to adding a new measurement value. To solve equation (4) for $x$. All non zero entries of $x$ correspond to the unobservable branches. Further approaches for observability analysis are described in [10].

\subsection{State Estimation}

The main goal of state estimation (SE) is the determination of a consistent system state. System state is defined as complex voltage at every single node. In transmission and sub-transmission systems SE based on weighted least square (WLS) approach is commonly used. The correlation between system state vector $x=\left[f_{2} \ldots f_{n} e_{1} \ldots e_{n}\right]$ and measurement vector $z$ is not linear since nodal voltages and power flows do not correlate linearly to each other. Thereby, $n$ is the total number of buses. The function $h$ defines the non linear network equations of each measured value as a function of the state vector. These measured values are subject to a measurement error $e$. 10, 11]

$$
z(x)=h(x)+e
$$

The estimation of the measurement error is determined in such a way that the sum of all weighted squared errors is minimized. The weighting matrix $R$ is a diagonal matrix of the measurement errors. The weighting is inversely proportional to the variance $\sigma_{i i}$ of measurement error.

$$
J(x)=\sum_{i=1}^{m} \frac{\left(z_{i}-h_{i}(x)\right)^{2}}{R_{i i}} \stackrel{!}{=} \min
$$

Equation (6) can be solved iteratively by GaussNewton approach, including the calculation of $H$, being the Jacobian matrix of $h$.

To increase redundancy nodes can be considered whose injection and load is zero. Nodal power of these nodes is with high probability zero, so variance of measurement error becomes very small. Therefore, solving equation 5 can have numerical problems. These numerical problems are considered by Hachtel's Augmented Matrix Approach (HAM). The numerical problems are considered by this approach with separation into constraints. 12

\subsection{Determination of Flexibility Potential}

Based on system state flexibility potential can be determined. Flexibility potential means all feasible set points of MV systems at the connection point to $\mathrm{HV}$ system without neglecting any network restrictions.

\subsubsection{Determination of Flexibility Potential based with Monte Carlo Simulation}

Determination of flexibility potential by random variation of energy supply and load is presented in [5]. The set points of flexible feeders and loads are randomly changed and it is checked by power flow calculation whether a set point of the distribution system can be found without violating any network restrictions. Furthermore, it is proven that the set of all feasible set points can be described as an area.

\subsubsection{Optimization Method}

Approaches for determination of flexibility potential based on Optimal Power Flow (OPF) and linear optimisation are presented in [4, [13] and [6]. Only the maximum and minimum active and reactive set points of analysed distribution system are determined. This reduces the number of calculations to be carried out. 
5 Implementation of the flexibility determination in real MV systems
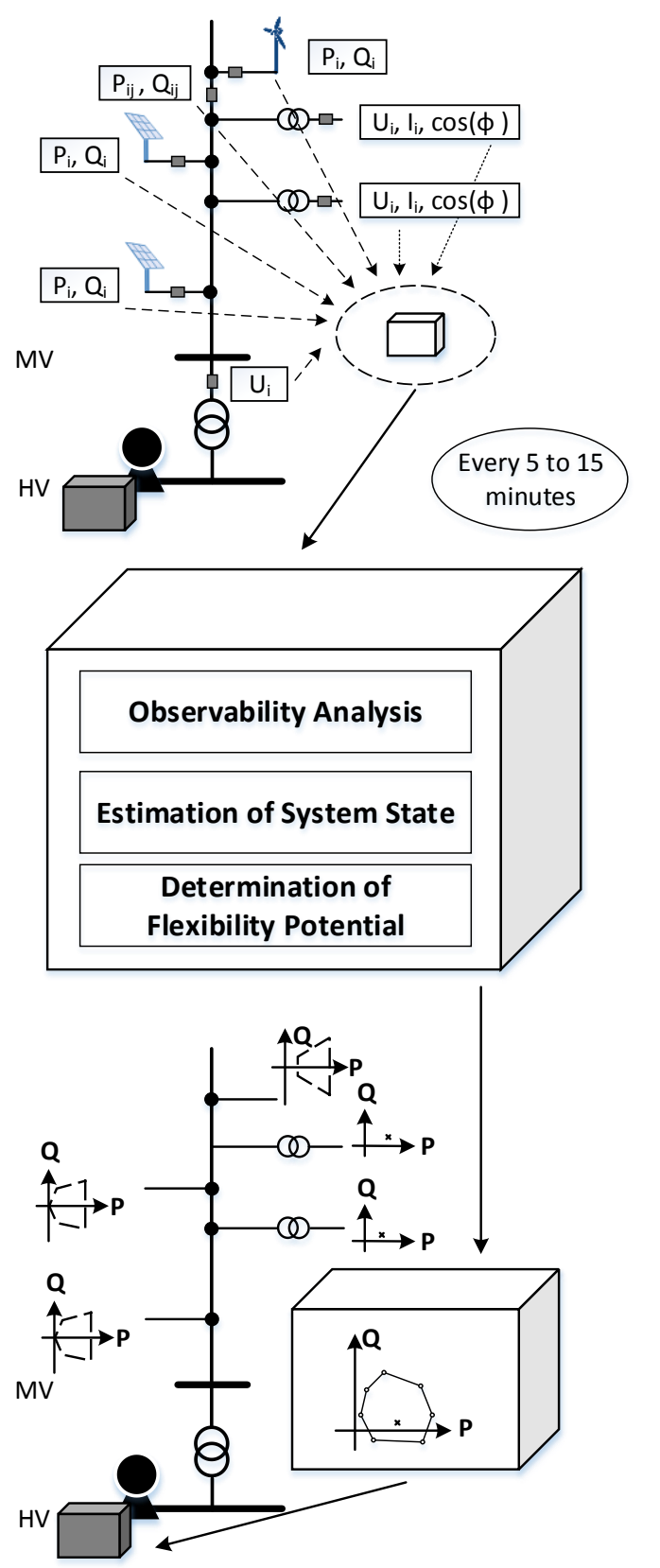

Figure 2: Overview field test

This section describe the implementation of SE and determination of flexibility potential in real MV system. The entire system is shown in figure (2).

\subsection{Measurement Devices}

To increase redundancy of measurement values and to test the developed methodology two MV systems are equipped with measurement devices as shown in figure (2). These measurement devices are installed in $\mathrm{MV} / \mathrm{LV}$ substations and measure three-phase voltage amount, current amount and power factor. In addition, the measured values of the DER connected to MV systems can be used. There are no power flow and phasor measurement unit (PMU) measurements currently in use. If available, these measurement values can also be used. The measured values are transmitted via Power Line Communication or wireless.

Depending on measurement devices measured values are averaged values over a period of five minutes. Otherwise, measured values are current values, where a minimum and maximum value is also specified within the period. Measured values of DER are current values without any further information. They are transmitted every 15 minutes.

\subsection{Determination of Flexibility Potential}

To determine the flexibility potential, it is first analysed whether the network topology can be fully observed with the available measured values. If the system is not observable, system state cannot be estimated and flexibility potential cannot be determined. The MV systems considered in the field test can be observed by the installed measurement technology and pseudo measurement values. Nevertheless, this can change if a measurement device or the communication network fails. In this case, further measured values can be added, pseudo measured values can be used or the network topology can be simplified. Observability analysis is only performed if there have been modifications in the measured value configuration or the network topology.

If the network is observable, SE can be executed and complex voltages can be determined on every single node. In the following step, complex nodal power is calculated using complex nodal voltages. These nodal voltages are input data to the algorithm for determining the flexibility potential with the known maximum and minimum active and reactive power limits of flexible generation units. No nodal power is calculated at the slack node. This nodal power is determined as flexibility potential during the next step. Flexibility potential is calculated by an optimization approach based on OPF and developed for this application. These algorithms have a modular structure so that different methods can be used for SE and determination of flexibility potential.

\subsection{Interface between HV and MV Network}

At the interface between HV and MV systems, the responsible agent is informed about the flexibility potential of analysed MV network. Not all feasible set points are communicated to the agent, but only boundary points of the area of flexibility. Information about system state and network topology are not exchanged. In cases of different HV and MV system operators, this exchange is not desired. The agent can activate the calculated flexibility potential if required. MV system operator modifies the set point of its system by itself on request by changing the set points of one or more flexible generation units. 


\subsection{Field Test}

The described approach will be tested over a period of six months in two MV networks. In these networks, certain parts are completely equipped with measurement devices. The test is over a period of six month in order to test the functionality of the algorithms and availability of communication technology on the one hand and to investigate flexibility at different seasons on the other hand. The measured values as well as the calculated system states and flexibility potentials are documented for research purposes. The presented system for determining flexibility potentials can also be used regardless of CMS. This could be cases which are described in section II.

During the field test only estimating of system state and determination of flexibility potential can be tested. Flexibility cannot be requested, so the set point of two MV systems cannot be changed. The components of HV system are tested by hardware-in-the-loop simulation in laboratory.

\section{Further Research Questions and Out- look}

This sections gives a short outlook to further research questions.

\subsection{Determination of Pseudo Measurement for non measuring substations}

Set points of substations with measurement devices are to be analysed and checked to what extent pseudo measurements values for stations without measurement technology can be obtained. This is intended to reduce the number and costs if measurement devices. Furthermore, an optimal placement of measurement devices can be determined from this data.

\subsection{Forecast of Flexibility Potential}

System operation of HV systems requires not only flexibility potential at the current point in time, but also for future points in time. The existing measurements values of different substations are therefore to be investigated for correlation with weather data.

\section{References}

[1] A. Primadianto and C. Lu, "A review on distribution system state estimation," IEEE Transactions on Power Systems, vol. 32, no. 5, pp. 3875-3883, 2017.

[2] J. Wu, Y. He, and N. Jenkins, "A robust state estimator for medium voltage distribution networks," IEEE Transactions on Power Systems, vol. 28, no. 2, pp. 1008-1016, 2013.

[3] R. Singh, B. C. Pal, and R. B. Vinter, "Measurement placement in distribution system state esti- mation," IEEE Transactions on Power Systems, vol. 24, no. 2, pp. 668-675, 2009.

[4] H. Chen and A. Moser, Eds., Improved flexibility of active distribution grid by remote control of renewable energy sources: 2017 6th International Conference on Clean Electrical Power (ICCEP), 2017.

[5] D. Mayorga Gonzalez, J. Hachenberger, J. Hinker, F. Rewald, U. Häger, C. Rehtanz, and J. Myrzik, "Determination of the time-dependent flexibility of active distribution networks to control their tsodso interconnection power flow," in 2018 Power Systems Computation Conference (PSCC), 2018, pp. 1-8.

[6] D. A. Contreras and K. Rudion, Eds., Improved Assessment of the Flexibility Range of Distribution Grids Using Linear Optimization: 2018 Power Systems Computation Conference (PSCC), 2018.

[7] B. Bauernschmitt, R. Palaniappan, D. Hilbrich, and C. Rehtanz, "Modular configurable and testable automation architecture for future active electrical energy grids: 2018 53rd international universities power engineering conference (upec)."

[8] C. Wagner, Integration und Bewertung der Spitzenkappung als Planungsgrundsatz zur wirtschaftlichen Netzentwicklung in Mittelspannungsnetzen, 1st ed., ser. Dortmunder Beiträge zu Energiesystemen, Energieeffizienz und Energiewirtschaft. Herzogenrath: Shaker, 2019, vol. 6 .

[9] O. Pohl, F. Rewald, S. Dalhues, P. Jörke, C. Rehtanz, C. Wietfeld, A. Kubis, R. K. Tamgue, and D. Kirsten, "Advancements in distributed power flow control," in 201853 rd International Universities Power Engineering Conference (UPEC), 2018.

[10] A. Abur and A. G. Exposito, Power System State Estimation: Theory and Implementation, ser. Power Engineering (Willis). Hoboken: Marcel Dekker Inc, 2004.

[11] E. Handschin, Elektrische Energieübertragungssysteme, 2nd ed., ser. ELTEX. Heidelberg: Hüthig, 1987.

[12] G. Bei, "Observability analysis for state estimation using hachtel's augmented matrix method," Electric Power Systems Research, vol. 77, no. 7, pp. 865-875, 2007.

[13] F. Capitanescu, Ed., TSO-DSO interaction: Active distribution network power chart for TSO ancillary services provision, vol. 163, 2018. 